Article type : Articles

\title{
Competitive ability, stress tolerance and plant interactions along stress
}

\section{gradients}

Man $\mathrm{Qi}^{1}$, Tao Sun ${ }^{1, *}$, SuFeng Xue ${ }^{1}$, Wei Yang $^{1}$, DongDong Shao ${ }^{1}$, and Javier MartínezLópez ${ }^{2}$

${ }^{1}$ State Key Laboratory of Water Environment Simulation, School of Environment, Beijing Normal University, Beijing 100875, China

${ }^{2}$ BC3-Basque Centre for Climate Change, Sede Building 1, 1st floor, Scientific Campus of the University of the Basque Country, Leioa 48940, Spain

Running Head: Testing the stress-gradient hypothesis

\begin{abstract}
Exceptions to the generality of the stress-gradient hypothesis (SGH) may be reconciled by considering species-specific traits and stress tolerance strategies. Studies have tested stress tolerance and competitive ability in mediating interaction outcomes, but few have incorporated this to predict how species interactions shift between competition and facilitation along stress gradients. We used field surveys, salt tolerance and competition experiments to develop a predictive model interspecific interaction shifts across salinity stress gradients. Field survey and greenhouse tolerance tests revealed trade-offs between stress tolerance and competitive ability. Modelling showed that along salinity gradients, 1) plant

${ }^{1 *}$ Correspondence author: suntao@bnu.edu.cn

This article has been accepted for publication and undergone full peer review but has not been through the copyediting, typesetting, pagination and proofreading process, which may lead to differences between this version and the Version of Record. Please cite this article as doi: $10.1002 /$ ecy. 2147

This article is protected by copyright. All rights reserved.
\end{abstract}


interactions shifted from competition to facilitation at high salinities within the physiological limits of salt-intolerant plants, 2) facilitation collapsed when salinity stress exceeded the physiological tolerance of salt-intolerant plants, and 3) neighbor removal experiments overestimate interspecific facilitation by including intraspecific effects. A community-level field experiment, suggested that 1) species interactions are competitive in benign and, facilitative in harsh condition, but fuzzy under medium environmental stress due to niche differences of species and weak stress amelioration, and 2) the SGH works on strong but not weak stress gradients, so SGH confusion arises when it is applied across questionable stress gradients. Our study clarifies how species interactions vary along stress gradients. Moving forward, focusing on SGH applications rather than exceptions on weak or nonexistent gradients would be most productive.

Key words: ecological theory; plant-plant interactions; process-based model; relative stress tolerance; salt marsh; stress-gradient hypothesis; plant community

\section{Introduction}

Understanding interspecific interactions is a fundamental goal of community ecology and essential for predicting how ecosystems respond to environmental change (Harmon et al. 2009, Harley 2011). Both positive (i.e. facilitation) and negative (i.e. competition) species interactions are included in ecological theory as important driving forces of community organization and dynamics (He and Bertness 2014). The stress-gradient hypothesis (SGH) (Bertness and Callaway 1994) predicts that competitive interactions switch to positive interactions with increasing biotic/abiotic stress due to amelioration of physical stress or associational consumer defenses. While the SGH has been widely supported (He et al. 2012, 2013), several questions remain (He and Bertness 2014), including: 1) whether competition 
can dominate in stressful conditions (Pennings et al. 2003, Schöb et al. 2013), and 2) whether facilitation collapses in extremely stressful conditions (Kawai and Tokeshi 2007, Holmgren and Scheffer 2010, Dangles et al. 2013). Since the SGH has been increasingly applied to models predicting plant population and community dynamics (Travis et al. 2006, Chu et al. 2008, 2009, 2010, Filotas et al. 2010, Wang et al. 2013), resolving these questions is crucial to refining predictions of how plant communities will respond to environmental change.

Controversies on the SGH have emerged because most empirical plant studies have focused on one or few pairwise species interactions, rather than community-level interactions Soliveres et al. 2015). Pairwise interactions can be primarily affected by species-specific traits, with environmental stress playing a secondary role (Soliveres et al. 2014, 2015). For example, stress tolerance, competitive ability (Liancourt et al. 2005, Graff and Aguiar, 2017), growth form, phylogeny, and life history stage (He et al. 2013) can mediate the outcome of plant interactions. Some have suggested that the SGH could be reconciled by incorporating the competitive ability (i.e., the ability to tolerate the inhibition of neighbors) and stress tolerance of the target species (Maestre et al. 2009, Soliveres et al. 2015). Interestingly, whole community-level studies of intertidal marine assemblages of seaweeds, marsh grasses and sessile and mobile invertebrates strongly supported the SGH (Altieri et al. 2007, Silliman et al. 2011, Crotty and Bertness 2015).

We generally do not know how far species-specific pairwise interactions can be extrapolated to the community-level since the predictability of the latter may depend on 1) whether stress in pairwise interactions is linked to the overall environmental conditions (Bertness and Callaway 1994, Bruno et al. 2003) and 2) whether there are multiple stresses on the community (Kawai and Tokeshi 2007, Michalet 2007). Increasing mechanistic understanding of pairwise interactions may improve insight to community-level interactions. 
Recent studies of pairwise interactions suggest that the distance to ecological optimum (the best performance of isolated individuals) of beneficiary species would determine the outcome of pairwise interactions (Liancourt et al. 2005). Thus, the further away a beneficiary species was from its optimum, the more important the nurse species would be. Moreover, when the performance of isolated individuals are linked to stress tolerances, evidence of the importance of the fundamental niche in determining pairwise interactions may appear. For instance, stress intolerant species were better facilitation candidates than stress-tolerators because they are more sensitive to stress alleviation (Liancourt et al. 2005, Graff and Aguiar 2017).

Competitive interactions under stressful conditions are frequently due to stress-tolerant species benefiting less from stress amelioration than stress intolerant species (Pennings et al. 2003). Thus, if we knew the shape of the stress tolerance curve, the competitive ability and amount of stress a benefactor can alleviate along stress gradients for co-occurring species, we could predict pairwise and community-level interactions shifts along stress gradients. These predictions could be to predict the effects of facilitation on community structure and function.

In this study, based on field survey and greenhouse experiments, we estimate the shape of the performance curve of tidal marsh plants along salinity gradients, the "Relative Stress Tolerance Curve (RSTC)". This curve was then implemented and tested in a dynamic competition model as the plant's competition strategy (Figure 1). The resulting quantitative predictions and a field transplant experiment were then analyzed to improve understanding 1) why and how stress intolerant species benefit more from stress amelioration; 2) how and when competition would shift to facilitation and 3) if facilitation collapses at extremely stressful conditions. 


\section{Methods}

\section{Study sites and species}

We chose the Yellow River Delta Nature Reserve $\left(37^{\circ} 35^{\prime}-38^{\circ} 12^{\prime} \mathrm{N}, 118^{\circ} 33^{\prime}-119^{\circ} 20^{\prime} \mathrm{E}\right)$ in the Shandong Province in China for this study. High elevation habitats, including uplands, terrestrial borders, and high marshes were used as study sites because the wide salinity gradient is the dominant abiotic stress (Cui et al. 2011). The transplant experiment and field surveys described below were conducted across this gradient. The three dominant species at the site and our target species were Phragmites australis, Tamarix chinensis, and Suaeda salsa. T. chinensis is a perennial shrub and its salt tolerance increases with age (Cui et al. 2010). Because it is difficult to conduct experiments that measure the exact stress tolerance and competitive ability of adult individuals of $T$. chinensis, we used one- to two-year-old juveniles of this species. A common invasive species $S$. alterniflora, which colonized mudflats, was included in the community-level field transplant experiment to increase the generality of our results. This species has a high salt tolerance similar to S. salsa (Qi et al. 2017). Variation in soil salinity at the site is driven by the groundwater table, evaporation, and precipitation with vegetation reducing soil evaporation and salinity stress by shading (Cui et al. 2011, He et al. 2012). (See Appendix S1 for site details).

\section{Field survey on plant distribution}

We quantified plant composition along the study site salinity gradient with eight transects (Fig. A1). In July 2015, we placed a $2 \times 2 \mathrm{~m}$ quadrat every $100 \mathrm{~m}$ along each transect (5 to 8 quadrats per transect) and recorded the percent cover of aboveground biomass of target species. Dry aboveground biomass was calculated by measuring the water content of the corresponding fresh aboveground plant tissue. Soil cores $(5.05 \mathrm{~cm}$ diameter $\times 5 \mathrm{~cm}$ deep) 
were collected at the center and four corners of each quadrat. Pore water salinity and moisture content was measured on these samples in the laboratory (see Qi et al. 2017).

\section{Greenhouse salt-tolerance tests}

We quantified salt-tolerance of $P$. australis, $T$. chinensis, and $S$. salsa in the greenhouse. Juveniles or ramets of each species were collected from monospecific areas at the study site and grown in a greenhouse over a wide range of salinities. We randomly excavated substrate blocks containing young ramets and juveniles from the field and transplanted sizestandardized ramet blocks $(21 \times 21 \times 18 \mathrm{~cm})$ of $P$. australis and $(18 \times 18 \times 15 \mathrm{~cm})$ blocks of S. salsa juvenile into pots. Three juveniles of T. chinensis were collected with intact substrate and planted in individual pots $(21 \times 21 \times 18 \mathrm{~cm})$. . . australis and $T$. chinensis aboveground biomass was cleared during transplantation to decrease transpiration and increase the survival of the juveniles. New buds or branches germinated within two weeks. We collected 25 replicates/species and grew them in the greenhouse for two weeks with fresh water for acclimation to greenhouse conditions before exposing them to salinity treatments. Plants exhibiting transplantation shock were discarded and replaced. Three replicates of each species were randomly assigned to seven salinity treatments ranging from 0 to 100 PSU (21 replicates/species). Salinity treatments were achieved by adding a salt solution at seven different concentrations $(0.5,2.0,5.0,10.0,15.0,30.0$, and 60.0 PSU) daily to maintain saturation and stable soil salinity. Plants were collected in late April 2014; salinity treatments were established on May 7 and maintained for four months.

Soil salinity (5 $\mathrm{cm}$ depth, measured as in the field survey) and plant performance (stem height, percentage of green/live plant tissue) of each replicate were monitored monthly. In September above- and belowground biomass of all replicates was harvested, dried, and weighed. Soil cores for water content and pore water salinity were also taken (Qi et al. 2017). Since the final dry biomass of replicates varied by as much as three orders of magnitude 
among species; the data were normalized for interspecific comparisons. For each species, we calculated the percent maximum biomass achieved by identifying the highest biomass replicate at the end of the salt tolerance experiments (Crain et al. 2004).

\section{Field transplant experiment}

To determine community-level interspecific interactions along a salinity gradient, we transplanted $P$. australis, T. chinensis, S. salsa and Spartina. alterniflora into upland, terrestrial border, and high marsh zones with and without neighbors of other species (Fig. A1). In mid-May 2014, we excavated substrate blocks $(10 \times 10 \times 20 \mathrm{~cm})$ containing healthy P. australis ramets, S. alterniflora ramets, S. salsa juveniles, and T. chinensis juveniles from nearby plant communities, and immediately transplanted them into upland, terrestrial border, and high marsh zones. At each transplant site (4 sites/habitat), we established five $0.5 \times 0.5 \mathrm{~m}$ plots all separated by at least $0.5 \mathrm{~m}$ from each other. At each site we planted the four species separately (four patches) and one mixed plot with all species (five different patches in total).

In each plot, transplants were evenly spaced, with the total number of substrate blocks and shrub juveniles kept at 25 to control plant density and competition. Transplants were watered daily for one week to reduce transplant shock. Transplant stem height and pore-water salinity were monitored monthly, before aboveground biomass was harvested and dried and weighed in mid-October 2014.

\section{Field plant removal experiments}

We conducted a plant removal experiment along the salinity gradient in April 2014 to measure salinity alleviation resulting from aboveground shading. We established three sites separated by $>50 \mathrm{~m}$ in the upland (least saline zone), terrestrial border (mediate saline zone), and the high marsh (most saline zone) where vegetation shading was observed to play a role in generating positive interactions among plants (He et al. 2012). In the three sample sites in 
each zone we established two $1 \times 1 \mathrm{~m}$ plots: a control plot and a plant removal plot, separated by $>2 \mathrm{~m}$. Aboveground biomass was removed biweekly as needed in the experimental treatments. A soil core $(5.05 \mathrm{~cm}$ diameter $\times 5 \mathrm{~cm}$ depth $)$ was taken in each plot monthly from April to July 2014 to determine pore-water salinity. Fresh and dry weights of aboveground vegetation were measured in each control plot in late July when plants achieved maximum biomass. Regressions were performed to quantified the relationship between soil salinity and salt stress alleviation ability and results were incorporated into the vegetation dynamic model below as plant-soil feedbacks.

\section{Model development}

A Stress Gradient Interspecific Interaction Model (SGIIM) was developed to link the competition and stress amelioration processes to elucidate the dynamic interplay between these processes. This model was kept as simple as possible while still containing the important processes discussed above. STELLA version 9.0 (Isee Systems, Inc.; Costanza and Voinov 2001) was used as a platform for our model (see Appendix S2: Fig. S1 for a model diagram). Parameters and equations used in the model (Appendix S2: Tables S1-S2) were acquired from our experiments described above and previous work (Qi et al. 2016; see Fig. 1 for a conceptual study flow diagram).

Salt-tolerance tests and the field survey were used to quantify target species RSTC later used in a competition model as the plant's competition strategy. RSTC can be represented as

$$
S_{i}=c_{i} \exp \left[-0.5\left(s_{t}-m_{i}\right)^{2} / r_{i}\right]
$$

where $S_{i}$ is the relative stress tolerance of species $i, c_{i}$ is a constant $\in[0,1]$ that represents the competitive ability of species $i$ at its fundamental niche optima; $\exp \left[-0.5\left(s_{t}-m_{i}\right)^{2} / r_{i}\right]$ is the standardized Gaussian stress tolerance curve; $s_{t}$ represents the stress intensity to which the species is exposed; $m_{i}$ is the most suitable environmental condition of species $i$; and $r_{i}$ is a 
constant reflecting the stress tolerance range of species $i$. This formula can represent variation in species competitive advantage across a stress gradient (as explained in Fig. 2).

A finite-difference equation was used to compute the change in plant population density, represented as dry aboveground biomass $\left(\mathrm{g} / \mathrm{m}^{2}\right)$ (Pacala and Tilman 1994):

$$
\Delta X_{i}=-d_{i} X_{i, t}+\left[\left(b i o_{\max }-\sum_{j} X_{j, t}\right)+\sum_{j} d_{j} X_{j, t}\right] P_{i}
$$

where $\Delta X_{i}$ is the change in population density of species $i$ from time $t$ to the next time step ( $t$ $+1), j$ represents all species in the community, and $X_{i, t}$ is the population density of $i$ at time $t$ $\left(\mathrm{g} / \mathrm{m}^{2}\right)$. The mortality rate of species $i$ is represented by $d_{i}$. bio $\max$ is the maximum environment carrying capacity within a plant community $\left(\mathrm{g} / \mathrm{m}^{2}\right)$, and $\sum_{j} X_{j, t}$ represents the total biomass of plant community at time $t . \sum_{j}^{\sum_{j} X_{j, t}}$ is the total decreased population density at time t. Thus, $\left[\left(b i o_{\max }-\sum_{j} X_{j, t}\right)+\sum_{j} d_{j} X_{j, t}\right]$ is the remaining carrying capacity of the plant community at time $t$, and $P_{i}$ is the probability that the species $i$ juvenile can occupy the remaining carrying capacity of the plant community, being equal to the relative stress tolerance $S_{i}$. Finally, the mortality rate equals to ${ }^{1 /\left(b S_{i}\right)}$, being a constant equal to 5 (Qi et al. 2016). The plant removal experiments quantified the relationship between soil salinity alleviation and vegetation biomass, which was incorporated into the competition model to calculate changes in soil salinity caused by vegetation shading. Plant distribution field survey data was used to validate the model (see Appendix S2: Fig. S2). The field transplant experiment and plant competition model were ultimately used to test the interspecific interaction along the stress gradient.

Model inputs include initial salinity level and species biomass. A salinity gradient ranging from 0 to 120 PSU was used to represent the field salinity range and the initial biomass of 
each species was set to a low level $\left(50 \mathrm{~g} / \mathrm{m}^{2}\right)$. The model was run yearly for a 10 -year simulation to achieve equilibrium for all simulations described below.

\section{Model-based interspecific interaction analysis}

Both pairwise plant interaction and community-level interaction were simulated to study variation in competition and facilitation. The model firstly simulated pairwise plant interactions between two spatially adjacent species ( $P$. australis vs. $T$. chinensis and $T$. chinensis vs. S. salsa) across the salinity gradient with and without neighboring species, with and without salinity amelioration feedback. To study the interspecific interaction mechanism, we used competition between $T$. chinensis and $S$. salsa as an example, and selected $T$. chinensis as a target species to analyzed its biomass distribution under four different scenarios, i.e. species grow (1) without neighboring species and salinity amelioration process (biomass distribution curve was set as $b^{T . c}$ ), (2) without neighboring species but with salinity amelioration process (biomass distribution curve was set as $b_{f}^{T . c}=b^{T . c}+\xi_{f}^{T . c}$ for easier comparison with scenario (1)), (3) with neighboring species but without salinity amelioration process (biomass distribution curve was set as $b_{c}^{T . c}=b^{T . c}+\xi_{c}^{v s S . s}$ ), (4) with neighboring species and with salinity amelioration process (biomass distribution curve was set as $b_{f c}^{T . c}=b^{T . c}+\xi_{c}^{v S S . s}+\xi_{f}^{T . c \& S . s}$ ). Thus, the interspecific interactions could be calculated with the difference in $T$. chinensis biomass between scenario (2) and scenario (4); i.e. $b_{f c}^{T . c}-b_{f}^{T . c}=\xi_{c}^{v s S . s}+\xi_{f}^{T . c \& S . s}-\xi_{f}^{T . c}$, where $\xi_{c}^{v s S . s}$ represented population loss of T. chinensis due to the competition of S. salsa, $\xi_{f}^{T . c \& S . s}-\xi_{f}^{T . c}$ represented population alteration of T. chinensis resulting from altered stress amelioration ability when $S$. salsa was present (hereafter was called interspecific facilitation). Since $\xi_{c}^{\text {vsS.s }}<0$, the positive or negative value of this equation 
depended on the difference between $\xi_{f}^{T . c \& S . s}$ and $\xi_{f}^{T . c}$. Interspecific interactions $\left(\xi_{c}^{v s j}+\xi_{f}^{i}-\xi_{f}^{i \& j}, i\right.$ is the target species, $j$ is the neighboring species) of pairwise interaction ( $P$. australis vs. T. chinensis and T. chinensis vs. S. salsa) were analyzed to see how interspecific interaction vary between benefactors and beneficiaries.

We then simulated community-level interspecific interaction among P. australis, $T$. chinensis, and S. salsa by comparing each species' distribution with and without neighbor species. Community-level simulations were compared with pairwise simulation and field transplant experiment results to test if pairwise interaction can be scaled up to community level, and how interspecific interaction change at community level.

\section{Results}

\section{Field survey and salt-tolerance tests}

The field survey (Fig. 3a) showed zonal distribution where P. australis occupied the upland with a narrow salinity range; $T$. chinensis dominated the terrestrial border with a broader salinity range; and $S$. salsa dominated the high marsh with the widest salinity range.

With above field distribution of species, and their salt tolerance result (Fig. 3b-d), we deduced their competitive abilities. Among species, S. salsa had the broadest salinity tolerance and a wide fundamental niche, as indicated by its large distribution range. Moreover, $S$. salsa reached its maximum growth at the lower end of the salinity gradient grown alone (Fig. 3d) and its lower abundance with neighboring species (Fig. 3a) revealed its low competitive ability. In contrast, $P$. australis was the most salt intolerant species but showed a high competitive ability, which allowed it to dominate the low salinity habitat. 
Interspecific interactions along salinity gradients: Field transplant experiments

Transplant experiments indicated that competition was strongest in upland habitats where all species can survive (Fig. 3e-h). With increasing soil salinity from upland to terrestrial border and high marsh (Appendix S3: Fig. S1), some salinity intolerant species, i.e. $P$. australis and T. chinensis, cannot survive at terrestrial border and/or high marsh, thus we did not observe a facilitative effect of neighboring species on these species. Yet we still found competitive effect of neighboring species on $T$. chinensis weakened with increasing salinity level. For species with a wide salinity tolerance range, i.e. S. salsa and S. alterniflora, we found neighboring species' effect shifted to facilitation at high marsh where the only surviving species were $S$. salsa and S. alterniflora. For each species, the location where facilitative effects of neighboring species varied depending on their specific niche width.

For each species, though location where facilitative effects of neighboring species differed regarding their specific niche width. It seems facilitation occurs near species' physiological limit, and community-level interspecific interaction shifted from competition at upland to facilitation at terrestrial border.

\section{Salinity alleviation ability: Field plant removal experiments}

Soil salinity alleviation was positively related to the aboveground biomass fresh weight and to substrate salinity (Appendix S3: Fig. S2): the regression expression was $Z=$ $1.2749 \ln (\mathrm{X}+1)(\exp (\mathrm{Y} / 80)-1), \mathrm{r}^{2}=0.9708$, where $\mathrm{Z}$ is the ameliorated salinity value (PSU), $\mathrm{X}$ is the fresh weight of aboveground plants $(\mathrm{g})$ and $\mathrm{Y}$ is the salinity of bare soil (PSU). It should be noted that we took use of this expression form to make sure ameliorated salinity value $(\mathrm{Z})$ equals to zero when there is no plants $(\mathrm{X}=0)$ or salinity of bare soil $(\mathrm{Y})$ is zero. It should be noted that this regression function is only validated when the range of $\mathrm{X}$ and $\mathrm{Y}$ is within the parameter space of the field data shown in dots. 


\section{Modelling pairwise interspecific interactions along salinity gradients}

When salinity amelioration was not considered, only interspecific competition occurred without facilitation (Fig. 4a, c). Competition intensity was high with low salt stress, and decreased to zero near the stress limit of stress intolerant species. More specifically, the competitive effect of relatively salt tolerant $T$. chinensis on salt-intolerant $P$. australis declined to zero at 30 PSU, a relative high salinity stress level before $P$. australis died at 40 PSU (its physiological salinity tolerance: Fig. 4a). Similarly, the competitive effect of $S$. salsa on $T$. chinensis decreased to zero at $40 \mathrm{PSU}$, a relatively high salinity stress before $T$. chinensis died at 60 PSU (its physiological salinity tolerance: Fig. 4c).

When the impact of salinity amelioration on interspecific interactions was considered, interspecific facilitation emerged at the upper part of the target species' physiological salinity tolerance, and peaked near the salinity level where competition intensity originally equaled to zero (Fig. 4b, d). In addition, though both interacting species were facilitated by the other, salinity intolerant species benefited more from neighboring species, and their realized distributions were extended, thus they were more likely to be benefactors. Using the coexistence of $T$. chinensis and $S$. salsa as an example (Fig. 5), we found that when $T$. chinensis grew in isolation (scenario (1) and (2), the salinity amelioration pushed the distribution curve $\left(b^{T . c}\right)$ to a higher salinity $\left({b^{T . c}}^{T} \xi_{f}^{T . c}\right)$, where $\xi_{f}^{T . c}<0$ at the lower end of distribution and $\xi_{f}^{T . c}>0$ at the higher end of distribution. When plant-soil feedback was neglected and there was competition from S. salsa (scenario (3), T. chinensis biomass decreased to $b^{T . c}+\xi_{c}^{\text {vsS.s }}$, where $\xi_{c}^{\text {vsS.s }}<0$ and was smallest at the distribution peak for $T$. chinensis and biggest at the distribution limit. Further, when plant-soil feedback was considered during competition with S. salsa (from scenario (3) to scenario (4)), the $T$. 
chinensis distribution curve shifted to the right $\left(b_{f c}^{T . c}=b^{T . c}+\xi_{c}^{v s S . s}+\xi_{f}^{T . c \& S . s}\right)$, where $\xi_{f}^{\text {T.c\&S.s }}<0$ at the lower end of distribution and $\xi_{f}^{\text {T.c\&S.s }}>0$ at the higher end of its distribution.

Analysis of interspecific interactions $\left(\xi_{c}^{v s j}+\xi_{f}^{i}-\xi_{f}^{i \& j}\right)$ indicate that $\xi_{f}^{i \& j}-\xi_{f}^{i}$ varied between beneficiaries and benefactors (Fig. 6). For beneficiaries, such as T. chinensis (Appendix S3: Fig. S3a) and $P$. australis (Appendix S3: Fig. S3c) interspecific facilitation (i.e. $\xi_{f}^{\text {T.c\&S.s }}-\xi_{f}^{T . c}$ ) was weak in benign conditions and was negative under certain salinity levels, but was never less than zero in harsh conditions. For benefactors, such as $S$. salsa (Appendix S3: Fig. S3b) and T. chinensis (Appendix S3: Fig. S3d), interspecific facilitation was positive and weak under benign, but negative and strong in moderately stressful conditions. Interspecific facilitation was weaker than competition in benign conditions and only surpassed interspecific competition when it was near zero under harsh growth conditions.

Simulation of community-level interspecific interactions indicated that community-level interspecific interaction are competitive at low salinity level (less than 25 PSU in Fig. 6b). With the increase of salinity level, community-level interspecific interaction become diverged: some species (salinity intolerant species) experience facilitative effect from neighbors, meanwhile other species (salinity tolerant species) experienced competitive effect. With further increase of salinity level (over 38 PSU in Fig. 6b), all species are facilitated by neighboring species, though species that is relative less tolerant to salinity stress ( $T$. chinensis) are facilitated more by salinity tolerant species ( $S$. salsa).

\section{Discussion}

Our experimental results demonstrate a trade-off between salinity tolerance and competitive ability, resulting in stress-intolerant species with a relative competitive ability that increased faster than stress-tolerant species in stressful condition due to stress 
amelioration. This explains why stress intolerant species benefit more from neighboring species at stressful conditions. Thus stress-intolerant species and stress-tolerant species are more likely to be beneficiaries and benefactors of facilitative interactions, respectively.

Pairwise and community simulations indicated that for beneficiary and benefactor, competition shifted to facilitation at the upper limit of their physiological salinity tolerance (Figure $4 \mathrm{~b}, \mathrm{~d}$ ). This was also found in our field transplant experiment: except for $P$. australis and $T$. chinensis whose salinity tolerance ranges were relative narrow and our transplant experiment did not capture facilitative interactions; neighbor facilitation with $S$. salsa and $S$. alterniflora occurred at the upper limit of their physiological salinity tolerance (Fig. 3e-h).

Model simulations also predicted a collapse of facilitation, but our field transplant experiment could not confirm this, probably due to the fact that more densely distributed transplant sites and more strictly controlled experimental condition are required to increase the resolution of salinity and minimize experimental errors.

\section{Trade-offs between competitive ability and stress tolerance}

Previous studies have shown that interspecific interactions depend on both the stress tolerance and competitive ability of interacting species, as well as the position of the species within their realized niches (Soliveres et al. 2015). Three main predictions regarding stress tolerance can be drawn from these studies: 1) stress-tolerant species are more likely to be benefactors, while stress-intolerant species are more often beneficiaries; 2) species with the strongest competitive responses are likely to benefit the most from facilitation when environmental stress is in the upper part of their physiological tolerance (Liancourt et al. 2005); 3) greater distance from a species environmental optimum (i.e. increasing 'strain') is positively correlated with the benefit a species experiences from interspecific interactions (Choler et al. 2001). Our study suggests that the first two predictions are due to trade-offs 
between salinity tolerance and competitive ability. The third prediction is still uncertain as interspecific interactions changed nonlinearly and facilitation collapses when environmental stress exceeds a species’ physiological tolerance limit (Fig. 4).

The trade-offs between salinity tolerance and competitive ability has also been reported in other salt marsh communities (Crain et al. 2004, Martínez-López et al. 2015) and has been long recognized (Grime 1974, Liancourt et al. 2005, Lind et al. 2013). Trade-off resulted in stress intolerant and tolerant species to more likely be beneficiaries and benefactors in facilitative interactions, respectively. In fact, it has been long recognized that stress intolerant species are more likely to be beneficiaries in facilitative interactions, although few studies have considered trade-offs between competitive ability and stress tolerance, as proposed by Grime's (1974) C-S-R theory, to explain this ecological pattern. Our study links Grime's CS-R theory with the SGH, helping understand why stress intolerant species are often beneficiaries, and stress tolerators are often benefactors in facilitative interactions.

\section{Collapse of facilitation in extreme salt stress conditions}

Our experiments tested the hypothesis that the importance and/or intensity of facilitative effects might decline under extreme environmental stress. For non-resource related stress, the collapse of facilitation is generally thought to be caused by a decrease in the facilitative effect of plant neighbors (Michalet et al. 2006, Maestre et al. 2009, Holmgren and Scheffer 2010, Michalet et al. 2014). For the two pairwise interactions tested in this study, we found that $P$. australis died at 40 PSU when coexisting with $T$. chinensis, and its interspecific facilitation declined at 30 PSU (Fig. 4b), where T. chinensis still ameliorate salinity greatly, which result in $400 \mathrm{~g} / \mathrm{m}^{2}$ increment of $T$. chinensis biomass production compared to that without salinity amelioration (see $\xi_{f}^{\text {T.c\&P.a }}$ in Appendix S3: Fig. S3d). Similarly, T. chinensis died at 70 PSU when coexisting with $S$. salsa, and facilitative effect of $S$. salsa on $T$. chinensis decreased at 
50 PSU (Fig. 4d), where $S$. salsa was still successful at ameliorating salinity stress (Appendix S3: Fig. S3b). Thus, the collapse of facilitation was because stress intolerant species could not survive under "moderate stress" even with intense stress amelioration, salinity stress still exceeded the physiological tolerance limit of the stress intolerant species and led to its mortality. This finding concurs with Brooker et al (2005) and Maestre et al. (2009).

\section{Overestimation of interspecific facilitation}

Current understanding of facilitation is generally based on neighbor removal experiments in the field, which might overestimate interspecific facilitation. In these neighbor removal experiments, aboveground parts of neighboring species are cleared, leaving target individuals surrounded by vacant soil and growing alone. The biomass of these target plants is compared with the control group to estimate the facilitation effect. In nature, vacant space released by neighbor removal might allow individuals of the same species to naturally colonize the surrounding area. Thus, removing neighbors around target species rather than replacing them with individuals of the same species might resulted in potential intraspecific facilitation be counted as interspecific facilitation, i.e., overestimation of interspecific facilitation.

In our simulations, we compared the scenario (2) (isolated and with feedback) and scenario

(4) (mixed and with feedback) to calculate the interspecific interaction between T. chinensis and S. salsa (Fig. 4). If we had followed the design of previous neighbor removal experiments, the biomass of target species in scenario (2) would be expected to shift leftward, i.e. a decrease in biomass. As a result, facilitation would be measured as being stronger than it actually was at the upper distribution limit, and the shift from competition to facilitation would occur in less stressful environments. Furthermore, this misleading result was similar to the observations from a neighbor removal experiment by Choler et al. (2001), who claimed that biotic interactions in target species were competitive on the left side of their distribution 
and facilitative on the right side of their distribution. Thus, we suggest a third treatment, neighbor replacement experiments, be added to estimate the degree of intraspecific interaction in case interspecific interaction was overestimated.

\section{Scaling-up to the community level}

Scaling up pairwise interspecific interactions to the community-level should be cautiously done, given multiple co-occurring stresses and diverse stress response curves. In single stress studies like ours, community-level interspecific interaction were mainly determined by the RSTC of the interacting species. Our community simulation (Fig. 6) and field transplant experiment (Fig. 3 e-h) indicated that all species were exposed to competition at low environmental stress (i.e. upland), and species that survived at high environmental stress (i.e. high marsh) were facilitated by neighboring species. However, at medium environmental stress (i.e. terrestrial border), interspecific interactions diverged. What are the mechanisms?

Regression analysis of soil -salinity -alleviation indicated that salinity alleviation is less sensitive to plant productivity at less stressful conditions (Appendix S3: Fig. S2). Moreover, at low environmental stress, community productivity was high and close to the carrying capacity of the habitat (Fig. 3a), thus the introduction of another species did not increase productivity greatly. Consequently, species within the community gained little biomass due to the introduction of another species, which would compete with existing species for resources. Thus, competition was quite common at low environmental stress. On the contrary, at high environmental stress, interspecific interaction was very weak and the introduction of another stress-tolerant species did not intensify competition but rather alleviated the stress. In addition, the surviving species were near their physiological tolerance limit and stress alleviation allowed them to grow better, thus interspecific interactions became facilitative (see the interaction between $S$. salsa and S. alterniflora at high marsh in Fig. 4g-h, and the interaction between S. salsa and T. chinensis when salinity range between 40 to 50 PSU in 
Fig. 6). Different from high environmental stress conditions, where almost all species are at their distribution limit, at medium environmental conditions, some species reached their distribution limit and were facilitated by other species, whereas others that were near their optimal condition were exposed to competition from other species (like the interaction between $S$. salsa and S. alterniflora at terrestrial border in Fig. 4g-h, and community simulation in Fig. 6 when salinity range between 25 to 40 PSU). Thus, the apparently fuzzy interspecific interaction at medium environmental stress was actually attributable to niche differences of the interacting species.

As a general rule in ecology, the SGH has been repeatedly validated with seaweeds, marsh grasses and sessile and invertebrates on strong intertidal gradients rocky shores, cobble beaches, sand dunes and salt marshes (Altieri et al. 2007, Silliman et al. 2011, Crotty and Bertness 2015), which are all habitats dominated by strong limiting factors that can be ameliorated. Whereas along weak gradients, our study shows that interspecific interaction might be fuzzy possibly because of 1) the niche difference of species within a community, 2) weak stress amelioration process compared with extreme conditions. We suggest that the SGH works on habitats dominated by strong gradients that can be ameliorated by coexisting species. In habitats without strong directional stress gradients the SGH doesn't work or gets fuzzy. Thus, much of the confusion of the SGH is when it is applied to communities not really on a stress gradient (He and Bertness 2014).

Variation in community-level interspecific interactions would be more complicated to predict with multiple stresses, because these stresses might interact and change in different directions. Even for the simplest circumstances where stress gradients vary in the same 
direction, species might be tolerant to one stress and intolerant to another stress, making it challenging to predict variation in species interactions as affected by environmental stress. In those case, it would be advisable to identify the main stress type and make predictions based on the main stress type.

To conclude, pairwise and community-level species interaction modelling showed that along the salinity gradients, 1) plant interactions shifted from competition to facilitation at relatively high salinities within the physiological tolerance limits of the salt-intolerant plants,

2) facilitation collapsed when salinity stress exceeded the physiological tolerance of saltintolerant plants, and 3) neighbor removal experiments may overestimate interspecific facilitation because intraspecific facilitation is included. Combining our modelling and community-level field experiment, we suggest that 1) community level species interactions are competition at benign conditions, and facilitation at harsh conditions, but are fuzzy at medium environmental stress, 2) the SGH works on strong gradient, and does not work on weak gradient, so much of the confusion of the SGH is when it is applied to communities not really on a stress gradient. It is important to refocusing SGH work on meaningful application rather than exceptions on weak or nonexistent gradients.

\section{Acknowledgements}

This work was supported by National Basic Research Program of China (973) (2013CB430402), the National Science Foundation for Innovative Research Group (No. 51421065), the National Natural Science Foundation of China (No. 51279007) and the Fundamental Research Funds for the Central Universities.

This article is protected by copyright. All rights reserved. 


\section{References}

Altieri, A. H., B. R. Silliman, and M. D. Bertness. 2007. Hierarchical organization via a facilitation cascade in intertidal cordgrass bed communities. American Naturalist 169:195206.

Bertness, M. D., and R. Callaway. 1994. Positive interactions in communities. Trends in Ecology \& Evolution 9:191-193.

Brooker, R., Z. Kikvidze, F. I. Pugnaire, R. M. Callaway, P. Choler, C. J. Lortie, and R. Michalet. 2005. The importance of importance. Oikos 109:63-70.

Bruno, J. F., J. J. Stachowicz, and M. D. Bertness. 2003. Inclusion of facilitation into ecological theory. Trends in Ecology \& Evolution 18:119-125.

Castanho, C. T., A. A. Oliveira, and P. I. Prado. 2012. The importance of plant life form on spatial associations along a subtropical coastal dune gradient. Journal of Vegetation Science 23:952-961.

Choler, P., R. Michalet, and R. M. Callaway. 2001. Facilitation and competition on gradients in alpine plant communities. Ecology 82:3295-3308.

Chu, C.-J., F. T. Maestre, S. Xiao, J. Weiner, Y.-S. Wang, Z.-H. Duan, and G. Wang. 2008. Balance between facilitation and resource competition determines biomass-density relationships in plant populations. Ecology Letters 11:1189-1197.

Chu, C.-J., J. Weiner, F. T. Maestre, Y.-S. Wang, C. Morris, S. Xiao, J.-L. Yuan, G.-Z. Du, and G. Wang. 2010. Effects of positive interactions, size symmetry of competition and abiotic stress on self-thinning in simulated plant populations. Annals of Botany 106:647652.

Chu, C.-J., J. Weiner, F. T. Maestre, S. Xiao, Y.-S. Wang, Q. Li, J.-L. Yuan, L.-Q. Zhao, Z.W. Ren, and G. Wang. 2009. Positive interactions can increase size inequality in plant populations. Journal of Ecology 97:1401-1407. 
Costanza, R., and A. Voinov. 2001. Modeling ecological and economic systems with STELLA: Part III. Ecological Modelling 143:1-7.

Crain, C. M., B. R. Silliman, S. L. Bertness, and M. D. Bertness. 2004. Physical and biotic drivers of plant distribution across estuarine salinity gradients. Ecology 85:2539-2549.

Crotty, S. M., and M. D. Bertness. 2015. Positive interactions expand habitat use and the realized niches of sympatric species. Ecology 96:2575-2582.

Cui, B. S., Q. A. He, and Y. A. An. 2011. Community structure and abiotic determinants of salt marsh plant zonation vary across topographic gradients. Estuaries and Coasts 34:459469.

Cui, B. S., Q. C. Yang, K. J. Zhang, X. S. Zhao, and Z. Y. You. 2010. Responses of saltcedar (Tamarix chinensis) to water table depth and soil salinity in the Yellow River Delta, China. Plant Ecology 209:279-290.

Dangles, O., M. Herrera, and F. Anthelme. 2013. Experimental support of the stress-gradient hypothesis in herbivore-herbivore interactions. New Phytologist 197:405-408.

Filotas, E., M. Grant, L. Parrott, and P. A. Rikvold. 2010. The effect of positive interactions on community structure in a multi-species metacommunity model along an environmental gradient. Ecological Modelling 221:885-894.

Graff, P., and M. R. Aguiar. 2017. Do species' strategies and type of stress predict net positive effects in an arid ecosystem? Ecology 98:794-806.

Grime, J. P. 1974. Vegetation classification by reference to strategies. Nature 250:26-31.

Harley, C. D. G. 2011. Climate change, keystone predation, and biodiversity loss. Science 334:1124-1127.

Harmon, J. P., N. A. Moran, and A. R. Ives. 2009. Species response to environmental change: Impacts of food web interactions and evolution. Science 323:1347-1350.

He, Q., and M. D. Bertness. 2014. Extreme stresses, niches, and positive species interactions 
along stress gradients. Ecology 95:1437-1443.

He, Q., M. D. Bertness, and A. H. Altieri. 2013. Global shifts towards positive species interactions with increasing environmental stress. Ecology Letters 16:695-706.

He, Q., B. S. Cui, M. D. Bertness, and Y. An. 2012. Testing the importance of plant strategies on facilitation using congeners in a coastal community. Ecology 93:2023-2029.

Holmgren, M., and M. Scheffer. 2010. Strong facilitation in mild environments: the stress gradient hypothesis revisited. Journal of Ecology 98:1269-1275.

Kawai, T., and M. Tokeshi. 2007. Testing the facilitation-competition paradigm under the stress-gradient hypothesis: decoupling multiple stress factors. Proceedings of the Royal Society of London B: Biological Sciences 274:2503-2508.

Liancourt, P., R. M. Callaway, and R. Michalet. 2005. Stress tolerance and competitiveresponse ability determine the outcome of biotic interactions. Ecology 86:1611-1618.

Lind, E. M., E. Borer, E. Seabloom, P. Adler, J. D. Bakker, D. M. Blumenthal, M. Crawley, K. Davies, J. Firn, D. S. Gruner, W. Stanley Harpole, Y. Hautier, H. Hillebrand, J. Knops, B. Melbourne, B. Mortensen, A. C. Risch, M. Schuetz, C. Stevens, and P. D. Wragg. 2013. Life-history constraints in grassland plant species: a growth-defence trade-off is the norm. Ecology Letters 16:513-521.

Maestre, F. T., R. M. Callaway, F. Valladares, and C. J. Lortie. 2009. Refining the stressgradient hypothesis for competition and facilitation in plant communities. Journal of Ecology 97:199-205.

Martínez-López, J., J. Martínez-Fernández, B. Naimi, M. F. Carreño, and M. A. Esteve. 2015. An open-source spatio-dynamic wetland model of plant community responses to hydrological pressures. Ecological Modelling 306: 326-333.

Michalet, R. 2007. Highlighting the multiple drivers of change in interactions along stress gradients. New Phytologist 173:3-6.

This article is protected by copyright. All rights reserved. 
Michalet, R., R. W. Brooker, L. A. Cavieres, Z. Kikvidze, C. J. Lortie, F. I. Pugnaire, A. Valiente-Banuet, and R. M. Callaway. 2006. Do biotic interactions shape both sides of the humped-back model of species richness in plant communities? Ecology Letters 9:767-773. Michalet, R., Y. Le Bagousse-Pinguet, J.-P. Maalouf, and C. J. Lortie. 2014. Two alternatives to the stress-gradient hypothesis at the edge of life: the collapse of facilitation and the switch from facilitation to competition. Journal of Vegetation Science 25:609-613.

Pacala, S. W., and D. Tilman. 1994. Limiting similarity in mechanistic and spatial models of plant competition in heterogeneous environments. American Naturalist 143:222-257.

Pennings, S. C., E. R. Selig, L. T. Houser, and M. D. Bertness. 2003. Geographic variation in positive and negative interactions among salt marsh plants. Ecology 84:1527-1538.

Qi, M., T. Sun, M. Zhan, and S. Xue. 2016. Simulating dynamic vegetation changes in a tidal restriction area with relative stress tolerance curves. Wetlands 36:31-43.

Qi, M., T. Sun, H. Zhang, M. Zhu, D. Shao, W. Yang, and A. Voinov. 2017. Maintaining natural salt barrens inhibit the spread of an invasive marsh grass. Ecosphere 8(10):e01982. 10.1002/ecs2.1982.

Schöb, C., C. Armas, and F. I. Pugnaire. 2013. Direct and indirect interactions co-determine species composition in nurse plant systems. Oikos 122:1371-1379.

Silliman, B. R., M. D. Bertness, A. H. Altieri, J. N. Griffin, M. C. Bazterrica, F. J. Hidalgo, C. M. Crain, and M. V. Reyna. 2011. Whole-Community Facilitation Regulates Biodiversity on Patagonian Rocky Shores. Plos One 6:e24502.

Soliveres, S., F. T. Maestre, M. A. Bowker, R. Torices, J. L. Quero, M. García-Gómez, O. Cabrera, A. P. Cea, D. Coaguila, D. J. Eldridge, C. I. Espinosa, F. Hemmings, J. J. Monerris, M. Tighe, M. Delgado-Baquerizo, C. Escolar, P. García-Palacios, B. Gozalo, V. Ochoa, J. Blones, M. Derak, W. Ghiloufi, J. R. Gutiérrez, R. M. Hernández, and Z. Noumi. 2014. Functional traits determine plant co-occurrence more than environment or 
evolutionary relatedness in global drylands. Perspectives in Plant Ecology, Evolution and Systematics 16:164-173.

Soliveres, S., C. Smit, and F. T. Maestre. 2015. Moving forward on facilitation research: response to changing environments and effects on the diversity, functioning and evolution of plant communities. Biological Reviews 90:297-313.

Travis, J. M. J., R. W. Brooker, E. J. Clark, and C. Dytham. 2006. The distribution of positive and negative species interactions across environmental gradients on a dual-lattice model. Journal of Theoretical Biology 241:896-902.

Wang, Y., Z. Yang, S. Zhou, J. Soininen, D. Ai, Y. Li, and C. Chu. 2013. The effect of positive interactions on temporal turnover of community composition along an environmental gradient. Plos One 8: 1-8.

\section{Figure captions.}

Figure 1. Conceptual diagram showing the integration of experiments (grey boxes) and GRIIM (elements within the dashed line). The conceptual diagram of GRIIM shows the main flows, state variables and functions. 'State variables' represent variables that get accumulated, 'flow' is an activity that changes the magnitude of a state variable, and 'functions' imposes an input or an output to GRIIM. Within GRIIM each plant species was subjected to the same dynamics.

Figure 2. Relative stress tolerance curve (RSTC) that effectively represents the varying competition relationship among species. For a given pairwise species interaction that presents tradeoffs between stress tolerance ability and competitive ability, i.e. one with a higher competitive ability but lower stress tolerance ability (species 1 , dashed lines), and the other with a lower competitive ability but higher stress tolerance ability (species 2, solid lines), when the standardized stress tolerance curve (gray curves) was multiplied by the competitive 
ability constant $c_{i}$, the resulting relative stress tolerance curve (dark curves) would represent the modified competitive ability across a stress gradient. Thus, species 1 would be a stronger competitor under benign conditions, yet losing its competitive advantage under stressful conditions.

Figure 3. Experimental results. (a) shows field plant distribution along the salinity gradient, (b) $\sim$ (d) show the standardized biomass of Phragmites australis, Tamarix chinensis, and Suaeda salsa respectively at varying levels of salinity, based on greenhouse performance. (e) $\sim$ (h) show biomass of transplanted species with and without neighboring species in the field transplant experiment. T1 to T8 represent the eight field transects from upland to low marsh, SM in brackets refers to the corresponding mean salinity value in units of PSU. Data are shown as mean \pm SE for $n=5-8$ quadrats per field line transect, $n=3$ replicates per greenhouse treatment, $n=4$ replicates per field transplant treatment. Salinity tolerance curves (solid lines) are represented by Gaussian function in (b), (c), and (d). Bars sharing a letter are not significantly different from one another in $(e) \sim(h)$.

Figure 4. Model variation in pairwise species interactions across salinity gradients in coastal salt marshes. The pairwise competition between Phragmites australis and Tamarix chinensis without (a), and with salinity amelioration process (b); the pairwise competition between $T$. chinensis and Suaeda salsa without (c) and with (d) salinity amelioration process. In each panel, the distribution of target species was simulated with and/or without the existence of neighboring species, and changes in biomass due to neighboring species occurrence was calculated. Legend: P. a: P. australis, T. $c:$ T. chinensis, and S. $s:$ S. salsa. Subpanels represent the enlarged detail in the shaded area of each panel.

Figure 5. Distribution of Tamarix chinensis under four scenarios: (1), without neighboring species and salinity amelioration process; (2), without neighboring species but with salinity amelioration process; (3), with neighboring species Suaeda salsa but without salinity 
amelioration process; (4), with neighboring species S. salsa and with salinity amelioration process. Gray shadow in the main panel indicates the difference between the red and blue curves.

Figure 6. Variation in community-level species interactions across salinity gradients. (a) compares the results of target species' distribution across salinity gradients with and without neighbors; changes in biomass due to neighboring species occurrence was calculated in (b). Legend: P. a: Phragmites australis, T. c: Tamarix chinensis, and S. s: Suaeda salsa.

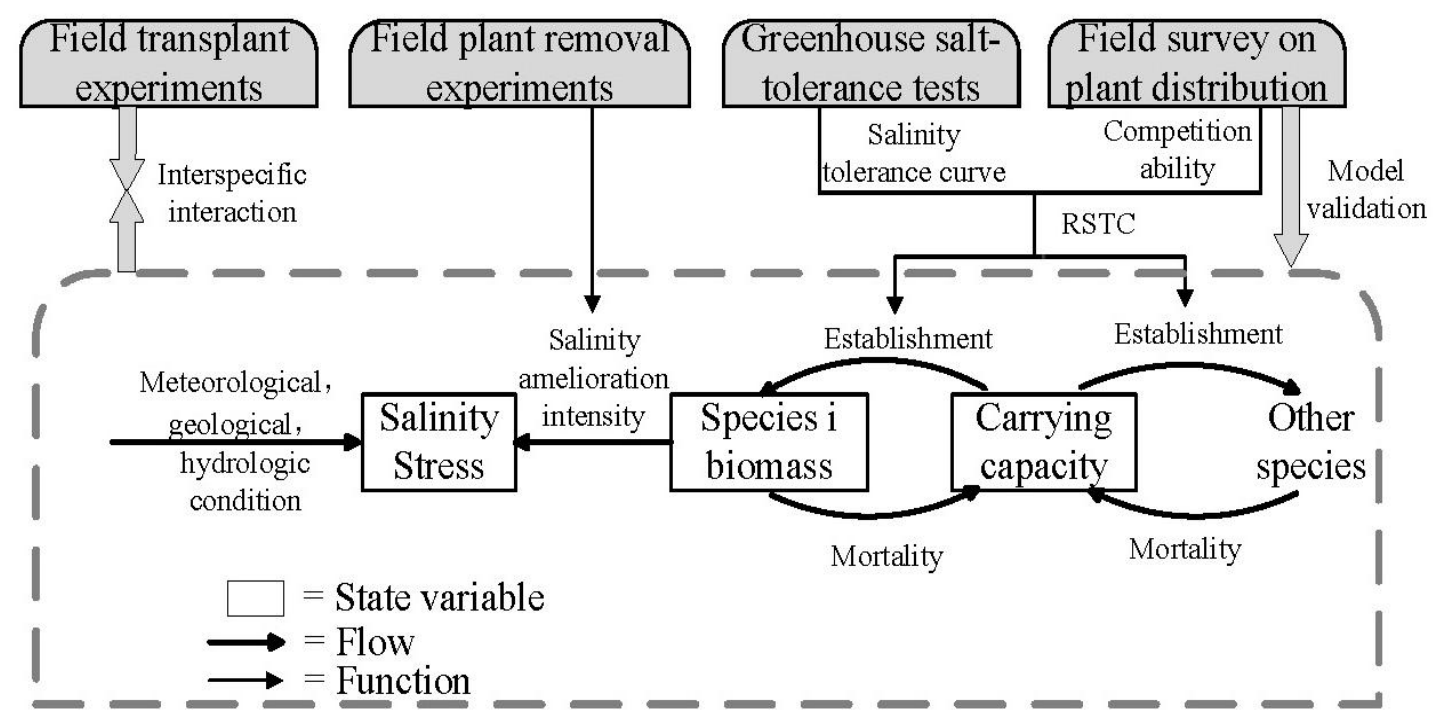

Figure 1.

This article is protected by copyright. All rights reserved. 


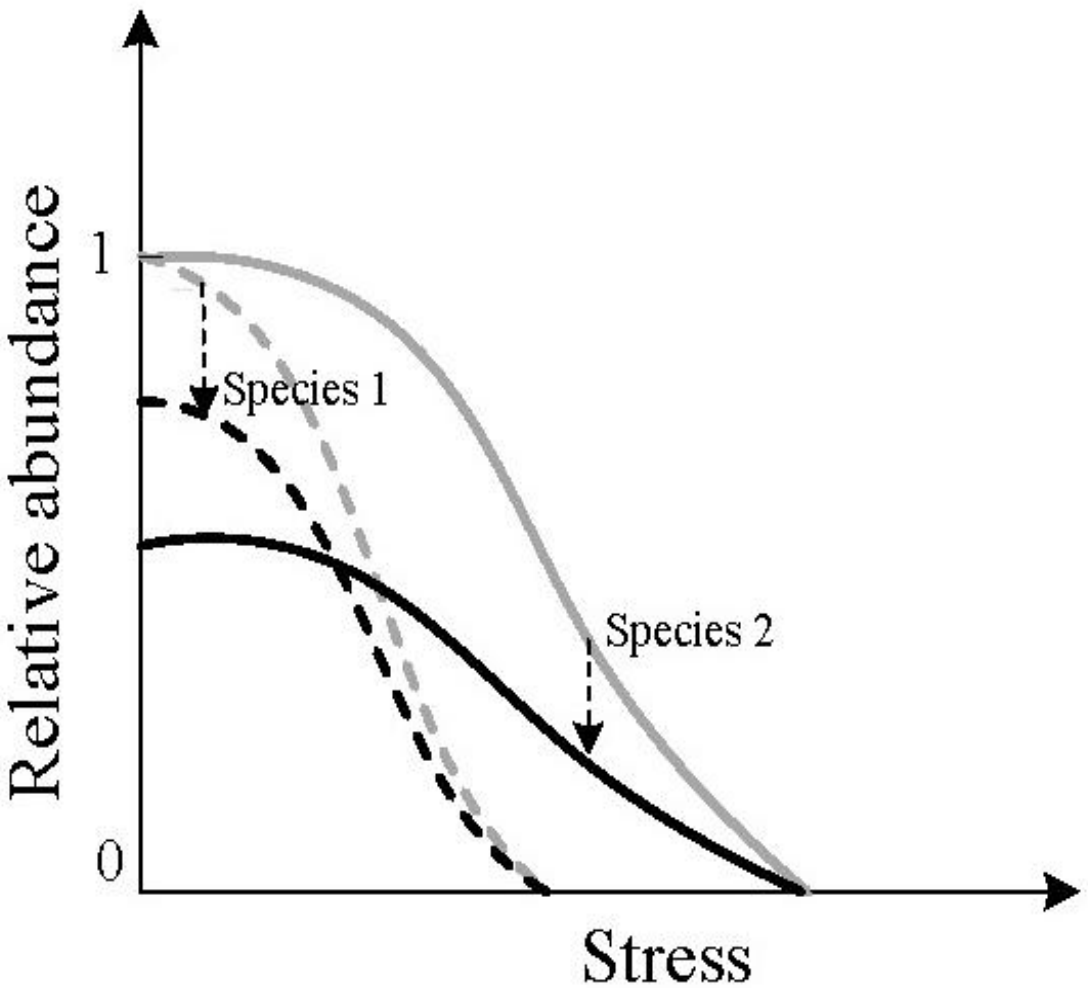

Figure 2. 

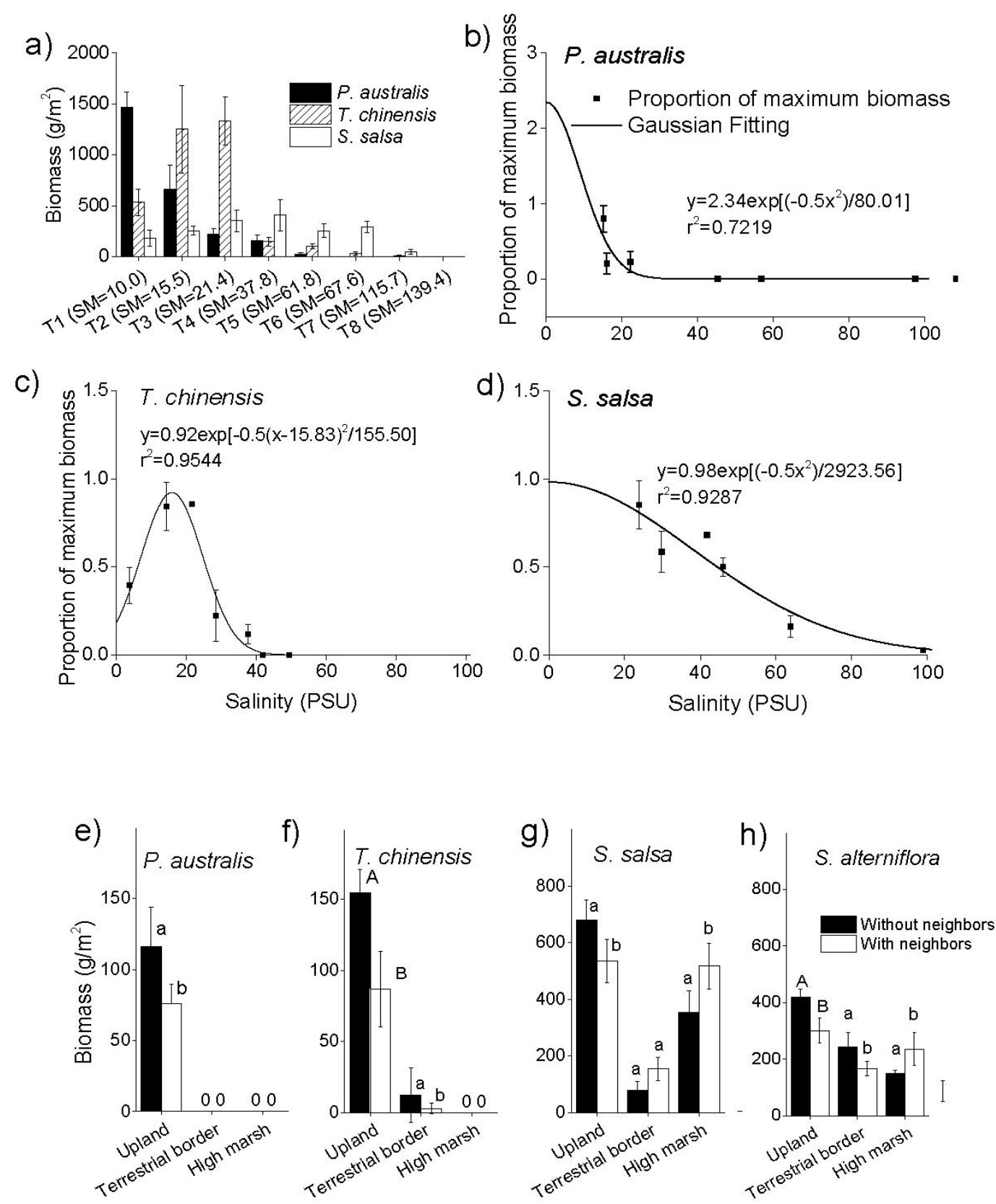

Transplant sites

Figure 3.

This article is protected by copyright. All rights reserved. 


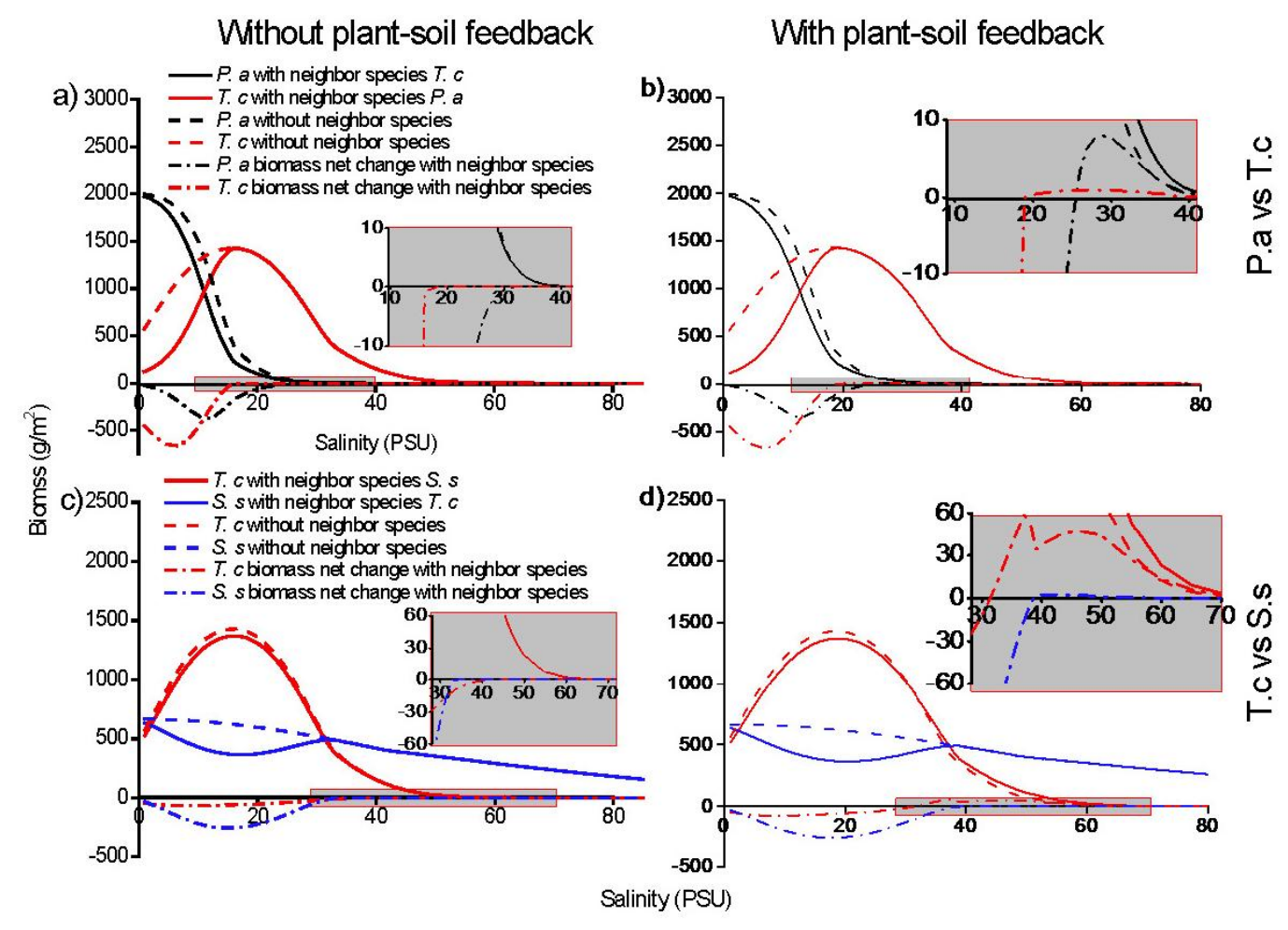

Figure 4.

This article is protected by copyright. All rights reserved. 


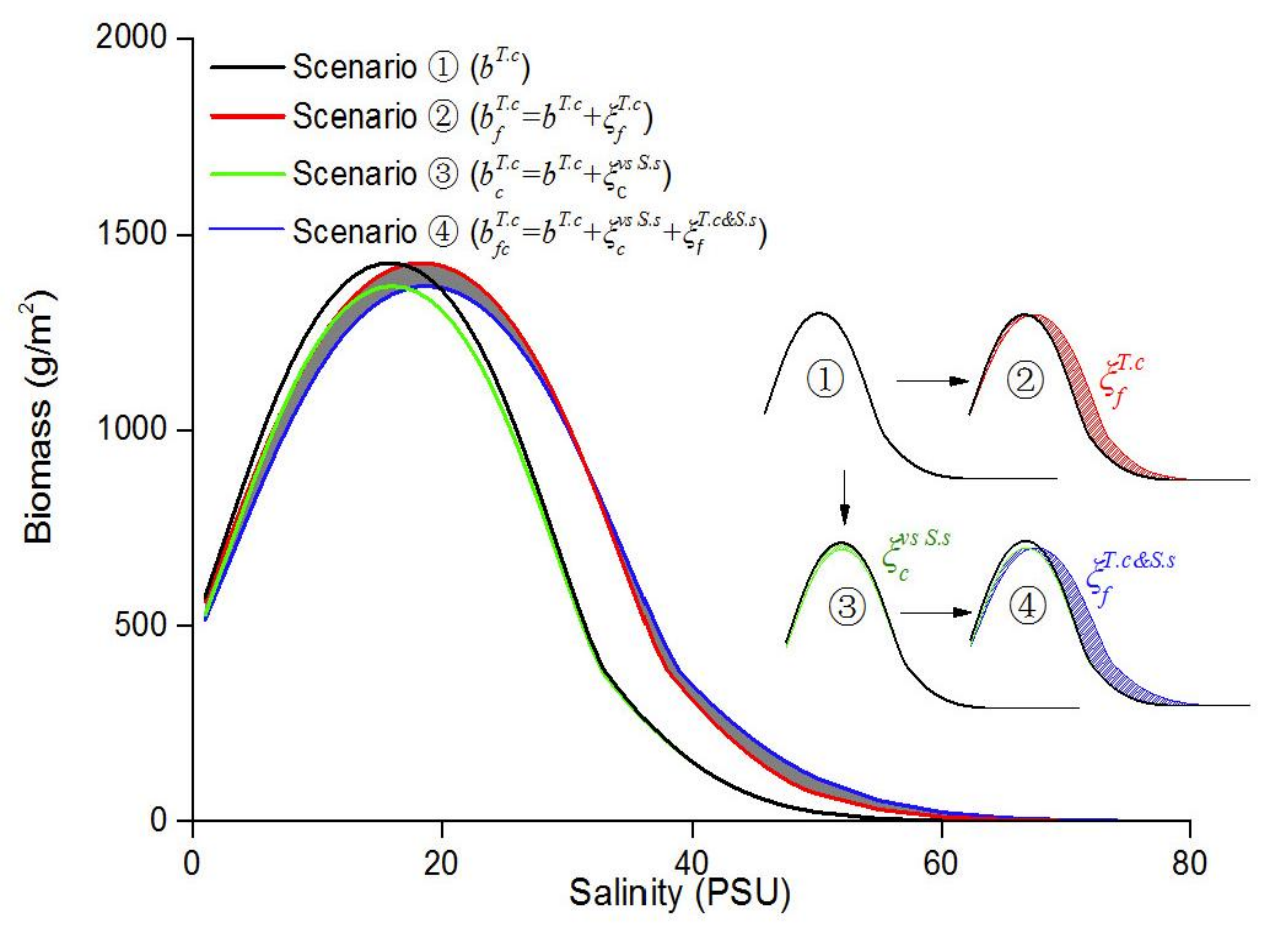

Figure 5.

This article is protected by copyright. All rights reserved. 

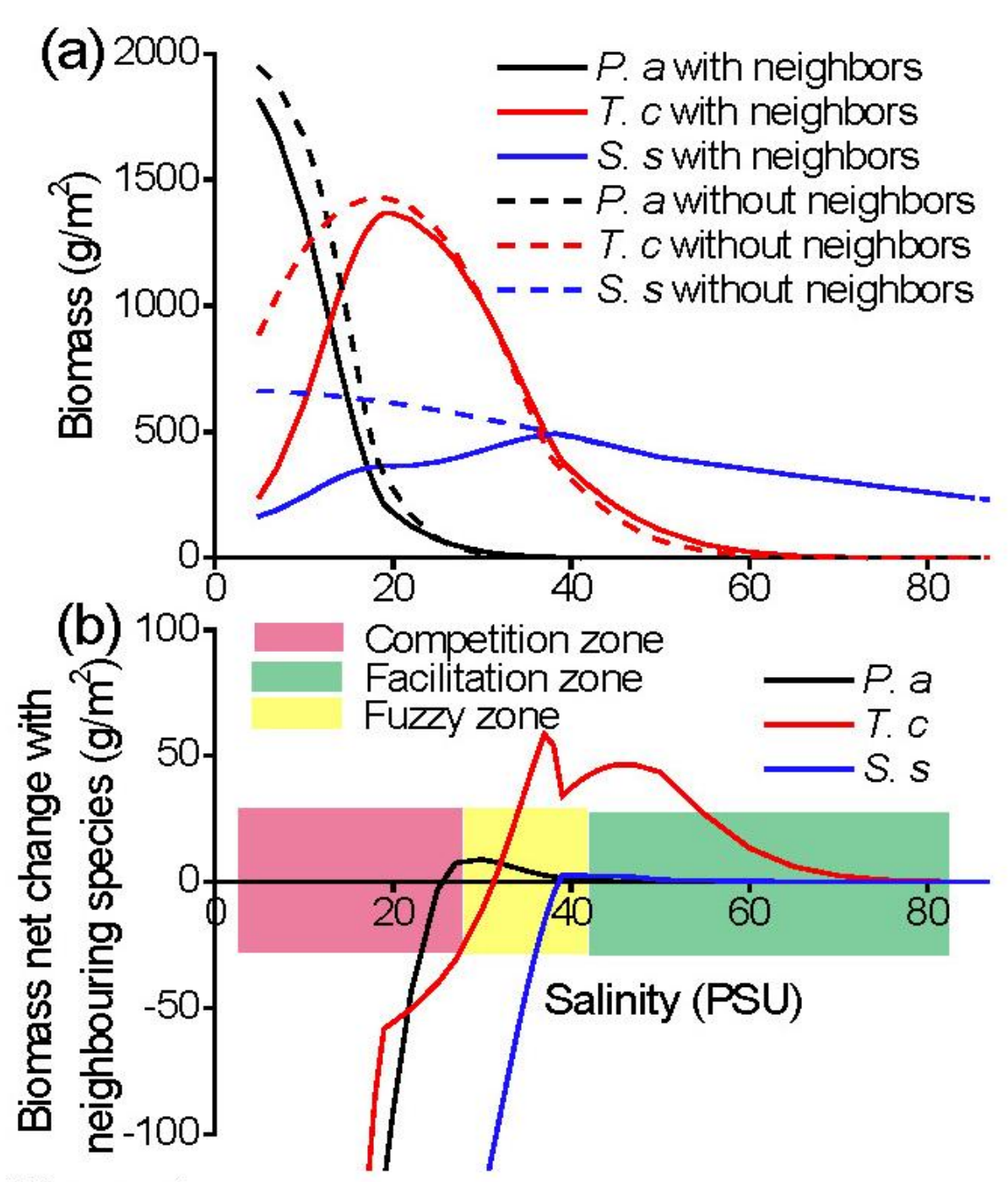

Figure 6. 